\title{
Surface Photoluminescence of Oxidized Nanodiamonds: Influence of Environment pH
}

Alexey M. Vervald ${ }^{1, *}$, Andrey V. Lachko ${ }^{1}$, Oleg S. Kudryavtsev ${ }^{2}$, Olga A. Shenderova ${ }^{3}$, Sergey V. Kuznetsov ${ }^{2}$, Igor I. Vlasov ${ }^{2}$, and Tatiana A. Dolenko ${ }^{1}$

${ }^{1}$ Faculty of Physics, M.V. Lomonosov Moscow State University, 1, bldg.2, Leninskie Gory, Moscow 119991, Russian Federation

${ }^{2}$ A.V. Prokhorov General Physics Institute of the Russian Academy of Sciences, 38, Vavilov Str., Moscow 119991, Russian Federation

${ }^{3}$ Adámas Nanotechnologies, Inc., 8100 Brownleigh Dr, Raleigh, NC 27617, USA

*Corresponding author, tel. and fax: +7 4959391104 ,

E-mail address: alexey.vervald@physics.msu.ru (A. Vervald)

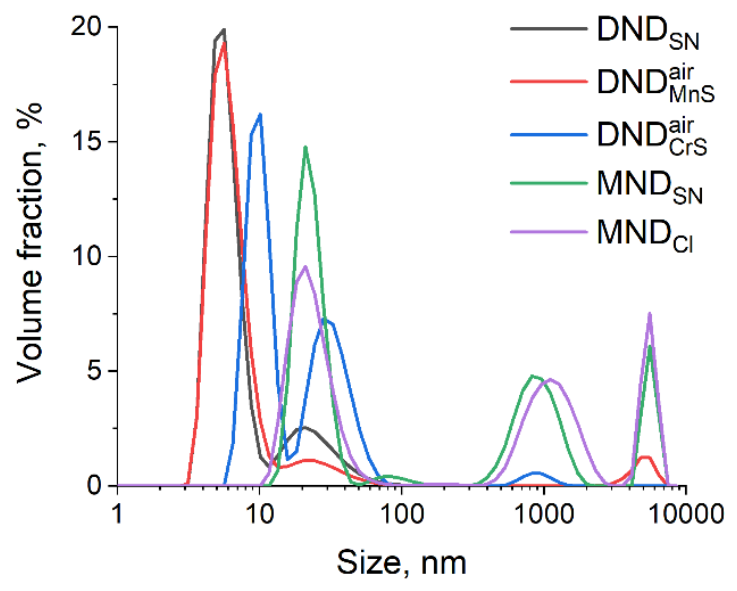

Figure S1. Distribution of nanodiamonds' aggregates volume fractions in aqueous suspensions.
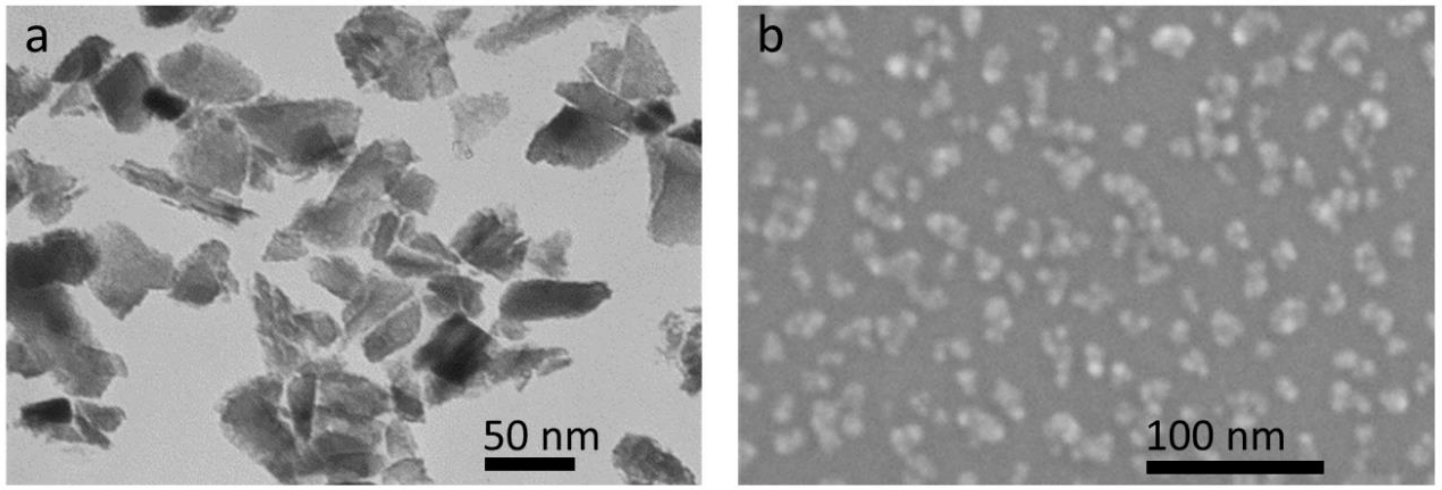

Figure S2. Electron microscopy images of unprocessed MNDs (a) and DNDs prior the fractionation (b) obtained on Carl Zeiss Libra 200 (60 kV, x150k) and Carl Zeiss Supra-25 $(20 \mathrm{kV}, \mathrm{x} 100 \mathrm{k})$, respectively. Please note that bigger nanoparticles are the clusters of the smaller ones, dispersion of which was diminished during drying of NDs over a substrate. 

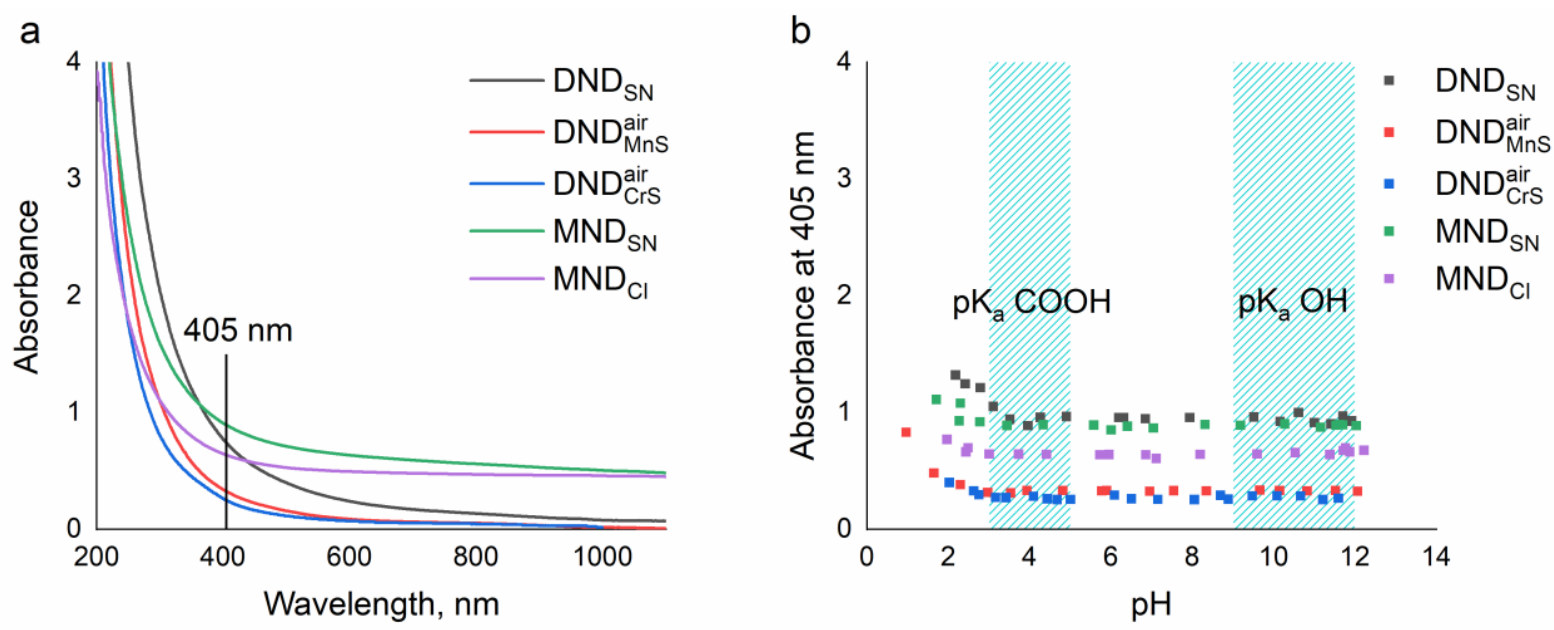

Figure S3. Absorbance spectra at neutral $\mathrm{pH}$ (a) and the absorbance at the wavelength of excitation $405 \mathrm{~nm}$ at different $\mathrm{pH}$ (b) of aqueous suspensions of NDs with concentration $0.5 \mathrm{~g} / \mathrm{L}$. 